\title{
SOME CHARACTERIZATIONS OF THE HEREDITARY PRETORSION CLASS OF SEMIGROUP AUTOMATA
}

\author{
by CLEMENT S. LAM
}

(Received 20 April, 1992)

\begin{abstract}
Let $S$ be a semigroup. A class of $S$-automata is called a hereditary pretorsion class (HPC) if it is closed under quotients, subautomata, coproducts (disjoint unions) and finite products. In this paper we present two characterizations of HPC. Specifically, we show that there is a bijective correspondence between the HPCs of $S$-automata, the right linear topologies on $S^{1}$ and the idempotent preradicals $\mathbf{r}$ on the category of $S$-automata such that the set of automata $\{M \mid \mathbf{r}(M)=M\}$ is closed under subautomata and finite products.
\end{abstract}

0. Introduction. In ring theory, there is an important correspondence between torsion modules, radicals on the category of ring modules and certain topologies on the ring. The general concept of torsion comes from the study of torsion modules in the rings of fractions and modules of fractions.

In this paper, we would like to begin the first step in obtaining similar results to semigroups and automata theories. We are able to establish the bijective correspondence between hereditary pretorsion classes of automata, right linear topologies on the semigroup adjoined with identity and certain idempotent preradicals.

Many people have studied radical and torsion theories in semigroups, monoids or their automata. For example, Marki, Mlitz and Strecker studied radicals and torsions on a collection of monoids in [3]. However, they defined the image of a radical as a congruence on the monoid while we defined the image of a preradical on an automaton as a subautomaton. On the other hand, Luedeman worked on torsion theories on monoids with zero and their automata in [2]. His approach was similar to ours but he had the advantage of defining the torsion theories on automata axiomatically as in the case of ring modules. He also made use of right ideals of monoids in his construction while we used right congruences as our main tool instead. The connection between right congruences and right ideals in rings is very intimate but it is not the case in semigroups. So we hope to present a different approach to those concepts here.

This paper is organized as follows: Section 1 provides some background definitons and preliminary results which can be found in [1] and [4]. Section 2 defines the right linear topologies on semigroups and on automata. Section 3 defines the hereditary pretorsion classes of automata and shows their correspondence with right linear topologies. Section 4 deals with preradicals on the category of automata and shows their connections with the hereditary pretorsion classes. The last section states the main correspondence theorem between the three concepts and also illustrates a particular example of a pretorsion class existence in the automata of fractions.

At this moment, the author would like to express his sincere gratitude to Professor Robert H. Oehmke for his advice on this project.

1. Basic definitions and the isomorphism theorem. Assume $S$ is a semigroup throughout this paper. 
Definition 1.1. A right congurence on $S$ is an equivalence relation $\rho$ on $S$ such that $(x, y) \in \rho$ and $s \in S$ imply $(x s, y s) \in \rho$.

Definition 1.2. $(M, S, \phi)$ is called an $S$-automaton if $M$ is a set, $S$ is a semigroup and $\phi: M \times S \rightarrow M$ is a function such that $\phi(\phi(m, s), t)=\phi(m, s t)$. For simplicity, we write $\phi(m, s)$ as $m s$ and $(M, S, \phi)$ as $M$.

Definition 1.3. Let $M$ be an $S$-automaton and $N$ a subset of $M$; then $N$ is called an $S$-subautomaton (or subautomaton) of $M$ if $N$ is closed under $\phi$; i.e. $n \in N$ and $s \in S$ imply $n s \in N$.

Definition 1.4. Let $M$ be an $S$-automaton. $\rho$ is a right congruence on $M$ if it is an equivalence relation on $M$ such that it is closed under the action by $S$; i.e. $(m, n) \in \rho$ and $s \in S$ imply $(m s, n s) \in \rho$.

Definition 1.5. Let $M$ be an $S$-automaton and $\rho$ a right congruence on $M$. Then $M / \rho$ is a set of equivalence classes of $\rho$. We define the action of the elements of $S$ on $M / \rho$ as follows: if $[m] \in M / \rho$ and $s \in S$, then $[m] s=[m s]$. This will turn $M / \rho$ into an $S$-automaton.

Definition 1.6. The direct product of a set of $S$-automata $\left\{M_{\alpha}\right\}$ is $\times M_{\alpha}$, where $S$ operates on $\times M_{\alpha}$ as follows: $\left(m_{\alpha}\right) s=\left(m_{\alpha} s\right)$ where $\left(m_{\alpha}\right) \in \times M_{\alpha}$.

Definition 1.7. The coproduct (or direct sum) of a set of $S$-automata $\left\{M_{\alpha}\right\}$ is the disjoint union $\oplus M_{\alpha}$, where $S$ operates on $\oplus M_{\alpha}$ in the obvious way.

Definition 1.8. A function $\alpha: M \rightarrow N$ between two $S$-automata $M$ and $N$ is called an $S$-homomorphism if it is closed under the action of $S$; i.e. $m \in M$ and $s \in S$ imply $\alpha(m s)=\alpha(m) s$.

Definition 1.9. The kernel $\operatorname{ker}(\alpha)$ of an $S$-homomorphism $\alpha: M \rightarrow N$ is the right congruence induced on $M$ by $\alpha$; i.e. $(m, n) \in \operatorname{ker}(\alpha)$ if and only if $\alpha(m)=\alpha(n)$.

Definition 1.10. Two $S$-automata $M$ and $N$ are said to be isomorphic if there is an $S$-homomorphism between $M$ and $N$ such that it is both injective and surjective.

THEOREM 1.1 (Isomorphism Theorem). If $\alpha: M \rightarrow N$ is a surjective $S$ homomorphism between two $S$-automata $M$ and $N$, then $M / \operatorname{ker}(\alpha) \cong N$.

Proof. Define $\Pi: M / \operatorname{ker}(\alpha) \rightarrow N$ by $\Pi([m])=\alpha(m)$.

For more basic results, please consult [1].

\section{Right linear (RL-) topologies on semigroups and on automata.}

DEFINITION 2.1. A topological semigroup is a semigroup $S$ with a topology such that the semigroup operation $(s, t) \mapsto s t$ is a continuous function from $S \times S$ to $S$ (considering the corresponding product topology on $S \times S$ ).

Definition 2.2. A topological semigroup $S$ is right linearly topological if there is a fundamental system of neighborhoods consisting of the equivalence classes of a set $\mathbf{T}$ of right congruences on $S$ such that this set $\mathbf{T}$ satisfies the following conditions.

T1. If $\rho \in \mathbf{T}, \sigma$ is a right congruence on $M$ and $\sigma \supset \rho$, then $\sigma \in \mathbf{T}$.

T2. If $\rho$ and $\sigma$ belong to $\mathbf{T}$, then $\rho \cap \sigma \in \mathbf{T}$.

T3. If $\rho \in \mathbf{T}$ and $u \in S$, then $(\rho: u) \in \mathbf{T}$, where

$$
(\rho: u)=\{(s, t) \in S \times S \mid(u s, u t) \in \rho\} .
$$


Definition 2.3. Let $\mathbf{T}$ be defined as above. A subset $G$ of $S$ is said to be open in $S$ if for each $a \in G$, there exists $\rho \in \mathbf{T}$ such that $[a]_{\rho}$ is contained in $G$. This defines a topology on $S$ and $\left\{[a]_{\rho} \mid a \in S, \rho \in \mathbf{T}\right\}$ is a basis. We will by abuse of language call $\mathbf{T}$ the topology for $S$.

LEMma 2.1. T makes the semigroup operation continuous.

Proof. Fix a point $(s, t) \in S \times S$. We show that given $\gamma \in \mathbf{T}$ with $[s t]_{\gamma}$, we can find $\alpha, \beta \in \mathbf{T}$ such that $a \in[s]_{\alpha}$ and $b \in[t]_{\beta}$ imply $a b \in[s t]_{\gamma}$. Let $\alpha=\gamma$; then $a \in[s]_{\alpha}$ implies that $a \in[s]_{\gamma}$, which implies that $(a, s) \in \gamma$ and then $(a b, s b) \in \gamma$. Next, since $\gamma \in \mathbf{T}$ and $s \in S,(\gamma: s) \in \mathbf{T}$ by T3. Let $\beta=(\gamma: s)$. Then $b \in[t]_{\beta}$ implies $(b, t) \in \beta$ which again implies $(s b, s t) \in \gamma$. By combining both results, we get $(a b, s t) \in \gamma$.

Next, let $S^{\prime}$ be $S$ adjoined with an identity element 1 if $S$ does not have an identity, otherwise it is equal to $S$. All automata in the following will be unitary. This means that the identity element of $S^{\prime}$ acts as the identity operator on the given automaton, where $S$ is the input semigroup of the automaton. Suppose $S^{\prime}$ is a right linearly topological semigroup with $\mathbf{T}$ as a neighborhood system of right congruences and $M$ is an $S$-automaton. We may consider $M$ as an $S^{1}$-automaton with the obvious modification.

Let $\mathbf{T}_{M}$ be a set of right congruences on $M$ satisfying the following conditions.

U1. If $\sigma$ is a right congruence on $M, \sigma \supset \rho$ and $\rho \in \mathbf{T}_{M}$, then $\sigma \in \mathbf{T}_{M}$.

U2. If $\rho$ and $\sigma$ belong to $\mathbf{T}_{M}$, then so does $\rho \cap \sigma$.

U3. If $\rho \in \mathbf{T}_{M}$ and $m \in M$ then $(\rho: m) \in \mathbf{T}$, where

$$
(\rho: m)=\left\{(s, t) \in S^{1} \times S^{1} \mid(m s, m t) \in \rho\right\} .
$$

This turns $M$ into a right linear topological $S$-automaton with the equivalence classes of elements in $\mathbf{T}_{M}$ as a neighborhood system. We will again by abuse of language call $\mathbf{T}_{M}$ a topology. In general, if $S$ is a right linear topological semigroup, then $M$ is a right linear topological $S$-automaton if it is an $S$-automaton, equipped with a topology consisting of right congruences on $M$ such that the map $M \times S \rightarrow M$, given by $(m, s) \mapsto m s$ is continuous. The construction here will be similar to that described as above for the right linear topological semigroup.

LEMMA 2.2. Let $S^{1}$ be a right linear topological semigroup with topology $\mathbf{T}$. For any $S$-automaton $M$, there is a topology on $M$, namely, the one for which the set of open right congruences is

$$
\mathbf{T}(M)=\{\rho / \rho \text { is a right congruence on } M \text { and }(\rho: x) \in \mathbf{T}, \forall x \in M\},
$$

where $(\rho: x)=\left\{(s, t) \in S^{1} \times S^{1} /(x s, x t) \in \rho\right\}$.

Proof. $\mathbf{T}(M) \neq \varnothing$ because it contains the universal congruence. Suppose $\sigma \supset \rho$ and $\rho \in \mathbf{T}(M)$. Let $x \in M$; then $(\sigma: x) \supset(\rho: x)$. Since $\rho \in \mathbf{T}(M),(\rho: x) \in \mathbf{T}$. Then $(\sigma: x) \in \mathbf{T}$ also by $\mathrm{T} 1$. Since this is true for all $x \in M, \sigma \in \mathbf{T}(M)$.

Next, suppose $\rho$ and $\sigma$ belong to $\mathbf{T}(M)$; then $(\rho: x) \in \mathbf{T}$ and $(\sigma: x) \in \mathbf{T}$. Then $(\rho: x) \cap(\sigma: x) \in \mathbf{T}$ by T2. However $(\rho \cap \sigma: x) \supset(\rho: x) \cap(\sigma, x)$. We conclude that $\rho \cap \sigma \in \mathbf{T}(M)$.

Finally, U3 is satisfied automatically by the definition of $\mathbf{T}(M)$.

LEMMA 2.3. Same notation as above. $\mathbf{T}(M)$ contains the trivial congruence if and only if $\operatorname{ker}\left(\alpha_{x}\right) \in \mathbf{T}, \forall x \in M$, where $\alpha_{x}: S^{1} \rightarrow x S^{1}$ is defined as $\alpha_{x}(s)=x s, \forall s \in S^{1}$. 
Proof. Suppose $\mathbf{T}(M)$ contains the trivial congruence $\iota$. Then, by definition, $(\iota: x) \in \mathbf{T}, \forall x \in M$. Now, $(s, t) \in(\iota: x)$ if and only if $(x s, x t) \in \iota$ if and only if $(s, t) \in$ $\operatorname{ker}\left(\alpha_{x}\right)$. We thus have $\operatorname{ker}\left(\alpha_{x}\right) \in \mathbf{T}, \forall x \in M$. The other direction can be proved by reversing all the previous steps.

Lemma 2.4. Suppose $M$ is an S-automaton and $x \in M$; then clearly $x S^{1}$ is an $S$-subautomaton of $M$. Moreover, $S^{1} / \operatorname{ker}\left(\alpha_{x}\right) \cong x S^{1}$, where $\alpha_{x}$ is as in Lemma 2.3.

Proof. As before, define $\alpha_{x}: S^{1} \rightarrow x S^{1}$ by $\alpha_{x}(s)=x s$. Both $S^{1}$ and $x S^{1}$ are $S$ automata. Furthermore, $\alpha_{x}(s t)=x(s t)=(x s) t=\alpha_{x}(s) t, \forall s, t \in S$. Therefore, $\alpha_{x}$ is an $S$-homomorphism and it is clear that $\alpha_{x}$ is surjective. Then $S^{1} / \operatorname{ker}\left(\alpha_{x}\right) \cong x S^{1}$ by the Isomorphism Theorem.

\section{Hereditary pretorsion classes of $S$-automata (HPC).}

Definition 3.1. A nonempty set of $S$-automata is called a pretorsion class if it is closed under quotients by right congruences, coproducts and finite products. We call its elements pretorsion $S$-automata.

Definition 3.2. A pretorsion class of $S$-automata is called hereditary if it is also closed under subautomata. We then call this set an HPC.

Theorem 3.1. Let $\mathbf{T}$ be an RL-topology on $S^{1}$. Define

$$
\mathbf{C}=\left\{M / M \text { is an } S \text {-automaton and } \operatorname{ker}\left(\alpha_{x}\right) \in \mathbf{T}, \forall x \in M\right\} .
$$

Then $\mathbf{C}$ is an $H P C$.

Proof. First of all, it is easy to show that $S^{1} / v \in \mathbf{C}$, where $v$ is the universal congruence. So $\mathbf{C} \neq \varnothing$. Next, suppose $M \in \mathbf{C}$ and $\rho$ is a right congruence on $M$. We show that $M / \rho \in \mathbf{C}$; i.e. $\mathbf{C}$ is closed under quotients. Let $x \in M$; then $[x] \in M / \rho$. Consider the $S$-homomorphism $\alpha_{|x|}: S^{1} \rightarrow[x] S^{1}$ given by $\alpha_{|x|}(s)=[x] s=[x s]$. Then $(s, t) \in \operatorname{ker}\left(\alpha_{|x|}\right)$ if and only if $[x s]=[x t]$. Therefore, $\operatorname{ker}\left(\alpha_{|x|}\right) \supset \operatorname{ker}\left(\alpha_{x}\right)$. Then $\operatorname{ker}\left(\alpha_{|x|}\right) \in \mathbf{T}$ by T1. Since $x \in M$ is arbitrary, we have $M / \rho \in \mathbf{C}$.

Next, it is clear that $\mathbf{C}$ is closed under coproducts and subautomata. We show that $\mathbf{C}$ is also closed under finite products. Let $M_{1}, \ldots, M_{n} \in \mathbf{C}$, and let $x=\left(x_{i}\right) \in M_{1} \times \ldots \times M_{n}$. Then

$$
\begin{aligned}
\operatorname{ker}\left(\alpha_{x}\right) & =\left\{(s, t) \in S^{1} \times S^{1} \mid \alpha_{x}(s)=\alpha_{x}(t)\right\} \\
& =\left\{(s, t) \mid x_{i} s=x_{i} t, \forall i=1, \ldots, n\right\} \\
& =\bigcap \operatorname{ker}\left(\alpha_{x_{i}}\right) .
\end{aligned}
$$

Since $M_{i} \in \mathbf{C}, \operatorname{ker}\left(\alpha_{x_{i}}\right) \in \mathbf{T}, \forall i=1, \ldots, n$, and hence $\operatorname{ker}\left(\alpha_{x}\right) \in \mathbf{T}$ by $\mathrm{T} 2$.

Theorem 3.2. Let $\mathbf{C}$ be an HPC. Define

$$
\mathbf{T}=\left\{\rho / \rho \text { is a right congruence of } S^{1} \text { and } S^{1} / \rho \in \mathbf{C}\right\} .
$$

Then $\mathbf{T}$ is an RL-topology on $S^{1}$.

Proof. Let $M \in \mathbf{C}$ and $x \in M$; then $x S^{1} \in \mathbf{C}$, since $\mathbf{C}$ is closed under subautomata. Since $x S^{1} \cong S^{1} / \operatorname{ker}\left(\alpha_{x}\right)$ by Lemma $2.4, \operatorname{ker}\left(\alpha_{x}\right) \in \mathbf{T}$. Thus, $\mathbf{T} \neq \varnothing$. Next, let $\sigma \supset \rho$ and 
$\rho \in \mathbf{T}$. Define $\eta: S^{1} / \rho \rightarrow S^{1} / \sigma$ by $\eta\left([s]_{\rho}\right)=[s]_{\sigma}$. Then it is easy to show that $\eta$ is a well-defined surjective $S$-homomorphism. By the Isomorphism Theorem, $\left(S^{1} / \rho\right) / \operatorname{ker}(\eta) \cong S^{1} / \sigma$. Since $\mathbf{C}$ is closed under quotient, $S^{1} / \sigma \in \mathbf{C}$ and hence $\sigma \in \mathbf{T}$. So T1 is established.

Now, suppose that $\rho$ and $\sigma$ belong to T. Consider a function $\theta: S^{1} / \rho \cap \sigma \rightarrow$ $S^{1} / \rho \times S^{1} / \sigma$ defined by $\theta\left([s]_{\rho \cap \sigma}\right)=\left([s]_{\rho},[s]_{\sigma}\right)$. Then, again, it is easy to show that $\theta$ is a well-defined $S$-homomorphism.

$$
\begin{aligned}
\operatorname{ker}(\theta) & =\left\{\left([s]_{\rho \cap \sigma},[t]_{\rho \cap \sigma}\right) \mid[s]_{\rho}=[t]_{\rho} \text { and }[s]_{\sigma}=[t]_{\sigma}\right\} \\
& =\left\{\left([s]_{\rho \cap \sigma},[t]_{\rho \cap \sigma}\right) \mid t \in[s]_{\rho} \cap[s]_{\sigma}=[s]_{\rho \cap \sigma}\right\} .
\end{aligned}
$$

Therefore $\operatorname{ker}(\theta)$ is the trivial congruence which implies that $S^{1} / \rho \times S^{1} / \sigma$ contains an isomorphic copy of $S^{1} / \rho \cap \sigma$. Hence, $S^{1} / \rho \cap \sigma \in \mathbf{C}, \rho \cap \sigma \in \mathbf{T}$ and T2 is established.

Finally, let $\rho \in \mathbf{T}$ and $s \in S^{1}$. Define $\beta: S^{1} \rightarrow S^{1} / \rho$ by $\beta(x)=[s x]_{\rho}$. Then $\beta(x t)=$ $[s(x t)]_{\rho}=[(s x) t]_{\rho}=[s x]_{\rho} t=\beta(x) t$ for $t \in S$. Therefore, $\beta$ is a well-defined $S$ homomorphism. Thus, $S^{1} / \rho$ contains an isomorphic copy of $S^{1} / \operatorname{ker}(\beta)$. Now

$$
\begin{aligned}
\operatorname{ker}(\beta) & =\left\{(x, y) \in S^{1} \times S^{1} \mid[s x]_{\rho}=[s y]_{\rho}\right\} \\
& =\{(x, y) \mid(s x, s y) \in \rho\} \\
& =\{(x, y) \mid(x, y) \in(\rho: s)\} .
\end{aligned}
$$

So $\operatorname{ker}(\beta)=(\rho: s)$. Thus, $S^{1} /(\rho: s) \in \mathbf{C}$ and hence $(\rho: s) \in \mathbf{T}$.

THEOREM 3.3. The set of hereditary pretorsion classes of $S$-automata can be parametrized by the set of right linear topologies on $S^{1}$.

Proof. To each $R L$-topology $\mathbf{T}$ we have associated an HPC, namely, $\mathbf{C}=\{M \mid M$ is an $S$-automaton and $\left.\operatorname{ker}\left(\alpha_{x}\right) \in \mathbf{T}, \forall x \in M\right\}$ by Theorem 3.1. Conversely, if $\mathbf{C}$ is an HPC, then $\mathbf{T}=\left\{\rho \mid \rho\right.$ is a right congruence on $S^{1}$ and $\left.S^{1} / \rho \in \mathbf{C}\right\}$ defines an $R L$-topology on $S^{1}$ by Theorem 3.2. It remains to verify that we have obtained a bijective correspondence between the set of HPC and the set of $R L$-topologies on $S^{1}$.

Let $\mathbf{T}$ be an $R L$-topology on $S^{1}$. Then we define $\mathbf{C}$ as above. Moreover, we define

$$
\mathbf{T}_{1}=\left\{\rho \mid \rho \text { is a right congruence on } S^{1} \text { and } S^{1} / \rho \in \mathbf{C}\right\}
$$

and

$$
\mathbf{T}_{2}=\left\{\rho \mid \rho \text { is right congruence on } S^{1} \text { and }(\rho: a) \in \mathbf{T}, \forall a \in S^{1}\right\} \text {. }
$$

Then we show $\mathbf{T}_{\mathbf{1}}=\mathbf{T}$ by proving that $\mathbf{T}_{\mathbf{1}}=\mathbf{T}_{\mathbf{2}}=\mathbf{T}$. First,

$$
\begin{aligned}
(\rho: s) & =\left\{(s, t) \in S^{1} \times S^{1} \mid(a s, a t) \in \rho\right\} \\
& =\left\{(s, t) \mid[a] s=[a] t \in S^{1} / \rho\right\} \\
& =\operatorname{ker}\left(\alpha_{[a]}\right) .
\end{aligned}
$$

It is, then, clear that $\mathbf{T}_{\mathbf{1}}=\mathbf{T}_{\mathbf{2}}$.

Next, let $\rho \in \mathbf{T}$ and $a \in S^{1}$; then $(\rho: a) \in \mathbf{T}$ by T3. Since this is true for all $a \in S^{1}$, $\rho \in \mathbf{T}_{\mathbf{2}}$. On the other hand, if $\rho \in \mathbf{T}_{\mathbf{2}}$, then $\rho=(\rho: 1) \in \mathbf{T}$. Therefore, we have established that $\mathbf{T}_{\mathbf{2}}=\mathbf{T}$. Hence $\mathbf{T}_{\mathbf{1}}=\mathbf{T}$.

Conversely, suppose $\mathbf{C}$ is an HPC. We define

$$
\mathbf{T}=\left\{\rho \mid \rho \text { is a right congruence on } S^{1} \text { and } S^{1} / \rho \in \mathbf{C}\right\} .
$$


Then define

$$
\mathbf{C}_{\mathbf{1}}=\left\{M \mid M \text { is an } S \text {-automaton and } \operatorname{ker}\left(\alpha_{x}\right) \in \mathbf{T}, \forall x \in M\right\}
$$

and

$$
\begin{aligned}
\mathbf{C}_{2}= & \{M \mid M \text { is an } S \text {-automaton and each cyclic subautomaton } \\
& \text { (i.e. } \left.\left.x S^{1} \text { where } x \in M\right) \text { is in } \mathbf{C}\right\} .
\end{aligned}
$$

We show that $\mathbf{C}_{\mathbf{1}}=\mathbf{C}$ by showing that $\mathbf{C}_{\mathbf{1}}=\mathbf{C}_{\mathbf{2}}=\mathbf{C}$.

Suppose $M \in \mathbf{C}_{\mathbf{l}}$; then $\operatorname{ker}\left(\alpha_{x}\right) \in \mathbf{T}, \forall x \in M$. Let $x \in M$; then $x S^{1}$ is a cyclic $S$-subautomaton of $M$. Since $x S^{1} \cong S^{1} / \operatorname{ker}\left(\alpha_{x}\right)$ and $\operatorname{ker}\left(\alpha_{x}\right) \in \mathbf{T}, x S^{1} \in \mathbf{C}$, and hence $M \in \mathbf{C}_{\mathbf{2}}$. Reversing the steps above, we establish $\mathbf{C}_{\mathbf{1}} \supset \mathbf{C}_{\mathbf{2}}$.

Next, if $M \in \mathbf{C}$, then obviously each cyclic subautomaton is also in $\mathbf{C}$, since $\mathbf{C}$ is closed under subautomata. Thus, $M \in \mathbf{C}_{2}$. Conversely, suppose $M \in \mathbf{C}_{2}$; then $x S^{\prime} \in \mathbf{C}$, $\forall x \in M$. Then $\oplus x S^{1} \in \mathbf{C}$. However then $M$ is the homomorphic image of $\oplus x S^{1}$ by the $S$-homomorphism which "forgets" the extra separating indices between the same elements in different cyclic subautomata. Hence $M \in \mathbf{C}$.

4. Preradicals on the category $\operatorname{Aut}(S)$ of $S$-automata. First note that the morphisms in $\operatorname{Aut}(S)$ are $S$-homomorphisms.

Definition 4.1. A preradical $\mathbf{r}$ is a functor on $\operatorname{Aut}(S)$ which assigns to each $S$-automaton $M$ a subautomaton $\mathbf{r}(M)$ in such a way that every $S$-homomorphism $\varphi: M \rightarrow N$ induces the map $\mathbf{r}(\varphi): \mathbf{r}(M) \rightarrow \mathbf{r}(N)$ by restriction.

Lemma 4.1. Suppose that $S^{1}$ is a right linearly topological semigroup with topology $\mathbf{T}$. For each $S$-automaton $M$, define

$$
\mathbf{t}(M)=\left\{x \in M / \operatorname{ker}\left(\alpha_{x}\right) \in \mathbf{T}\right\} .
$$

Then $\mathbf{t}$ is a preradical on $\operatorname{Aut}(S)$.

Proof. Suppose $M \in \operatorname{Aut}(S)$. Then clearly $M \supset \mathbf{t}(M)$. Let $x \in \mathbf{t}(M)$ and $u \in S$. Then $\operatorname{ker}\left(\boldsymbol{\alpha}_{x}\right) \in \mathbf{T}$ by definition. Now

$$
\begin{aligned}
\left(\operatorname{ker}\left(\alpha_{x}\right): u\right) & =\left\{(a, b) \in S^{1} \times S^{1} \mid(u a, u b) \in \operatorname{ker}\left(\alpha_{x}\right)\right\} \\
& =\{(a, b) \mid(x u) a=(x u) b\} \\
& =\operatorname{ker}\left(\alpha_{x u}\right) \in \mathbf{T} \text { by T3. }
\end{aligned}
$$

Thus $x u \in \mathbf{t}(M)$ and $\mathbf{t}(M)$ is an $S$-subautomaton of $M$.

Moreover, let $M$ and $N$ be $S$-automata and $\varphi: M \rightarrow N$ be an $S$-homomorphism. Consider the induced map $\mathbf{t}(\varphi): \mathbf{t}(M) \rightarrow N$ where $\mathbf{t}(\varphi)$ is equal to $\varphi$ restricted to $\mathbf{t}(M)$. Since $\operatorname{ker}\left(\alpha_{x}\right) \in \mathbf{T}, \forall x \in \mathbf{t}(M), \mathbf{T}(\mathbf{t}(M))$ contains the trivial congruence by Lemma 2.3 . Therefore $\operatorname{ker}(\mathbf{t}(\varphi)) \in \mathbf{T}(\mathbf{t}(M))$ by $\mathrm{U} 1$, and thus $(\operatorname{ker}(\mathbf{t}(\varphi)): x) \in \mathbf{T}, \forall x \in \mathbf{t}(M)$. However,

$$
\begin{aligned}
\operatorname{ker}\left(\alpha_{\mathbf{t}(\varphi)(x)}\right) & =\left\{(u, v) \in S^{1} \times S^{1} \mid \mathbf{t}(\varphi)(x) u=\mathbf{t}(\varphi)(x) v\right\} \\
& =\{(u, v) \mid(x u, x v) \in \operatorname{ker}(\mathbf{t}(\varphi))\} \\
& =(\operatorname{ker}(\mathbf{t}(\varphi)): x)
\end{aligned}
$$

Therefore, $\operatorname{ker}\left(\alpha_{\mathbf{t}(\varphi)(x)}\right) \in \mathbf{T}$ and hence $\mathbf{t}(\varphi)(x) \in \mathbf{t}(N)$. So the induced map is $\mathbf{t}(\varphi): \mathbf{t}(M) \rightarrow$ $\mathbf{t}(N)$ and we conclude that it is a preradical on $\operatorname{Aut}(S)$. 
Definition 4.2. A preradical $\mathbf{r}$ on $\operatorname{Aut}(S)$ is idempotent if $\mathbf{r}(\mathbf{r}(M))=\mathbf{r}(M)$ for all $M \in \operatorname{Aut}(S)$.

Lemma 4.2. The preradical $\mathbf{t}$ we defined in Lemma 4.1 is idempotent.

Proof. Clear.

Next, we would like to relate the preradical $\mathbf{t}$ with HPC.

Define $\mathbf{C}_{\mathbf{t}}=\{M \mid M$ is an $S$-automaton and $\mathbf{t}(M)=M\}$.

TheOREM 4.3. $C_{t}$ is an HPC.

Proof. $\mathbf{C}_{\mathbf{t}} \neq \varnothing$ since $\mathbf{t}$ is idempotent by Lemma 4.2. Suppose $M \in \mathbf{C}_{\mathbf{t}}$ and $N$ is an $S$-subautomaton of $M$. It suffices to show that $\mathbf{t}(N) \supset N$. Let $x \in N$; then $x \in M$ and $x \in \mathbf{t}(M)$ since $M=\mathbf{t}(M)$. But then $\operatorname{ker}\left(\alpha_{x}\right) \in \mathbf{T}$ by definition of $\mathbf{t}(M)$ and hence $x \in \mathbf{t}(N)$. So $\mathbf{C}_{\mathbf{t}}$ is closed under subautomata.

Secondly, suppose $M \in \mathbf{C}_{\mathbf{t}}$ and $\rho$ is a right congruence on $M$. We show that $M / \rho \in \mathbf{C}_{\mathbf{t}}$. Define $\pi: M \rightarrow M / \rho$ by $\pi(m)=[m]_{\rho}$. Then $\pi$ is a well-defined surjective $S$ homomorphism. Since $\mathbf{t}$ is a preradical, we have the induced map $\mathbf{t}(\pi): \mathbf{t}(M) \rightarrow \mathbf{t}(M / \rho)$. Since $M \in \mathbf{C}_{\mathbf{t}}$, the induced map is $\mathbf{t}(\pi): M \rightarrow \mathbf{t}(M / \rho)$. But $\mathbf{t}(\pi)(M)=\pi(M)=M / \rho$. Therefore $\mathbf{t}(M / \rho) \supset M / \rho$ and hence $M / \rho \in \mathbf{C}_{\mathbf{t}}$.

Thirdly, suppose $\left\{M_{\alpha}\right\}$ is a collection of $S$-automata in $\mathbf{C}_{\mathbf{t}}$. Since $\mathbf{t}\left(M_{\alpha}\right)=M_{\alpha}, \forall \alpha$, the induced map of each inclusion is $\mathbf{t}\left(M_{\alpha}\right) \rightarrow \mathbf{t}\left(\oplus M_{\alpha}\right)$ which is then $M_{\alpha} \rightarrow \mathbf{t}\left(\oplus M_{\alpha}\right)$. It follows that $\mathbf{t}\left(\oplus M_{\alpha}\right)=\oplus M_{\alpha}$ by the universal mapping property of coproducts. Hence $\mathbf{C}_{\mathbf{t}}$ is closed under coproducts.

Finally, we show that $\mathbf{C}_{\mathbf{t}}$ is closed under finite products. Suppose $M_{1}, \ldots, M_{n} \in \mathbf{C}_{\mathbf{i}}$; then $\mathbf{t}\left(M_{1} \times \ldots \times M_{n}\right)=\left\{x=\left(x_{i}\right) \in M_{1} \times \ldots \times M_{n} \mid \operatorname{ker}\left(\alpha_{x}\right) \in \mathbf{T}\right.$. Let $x=\left(x_{i}\right) \in M_{1} \times \ldots \times$ $M_{n}$. As before, $\operatorname{ker}\left(\alpha_{x}\right)=\bigcap \operatorname{ker}\left(\alpha_{x_{i}}\right)$. By hypothesis, $M_{i} \in \mathbf{C}_{\mathbf{t}}$. So $\mathbf{t}\left(M_{i}\right)=M_{i}, \forall i=$ $1, \ldots, n$, and then $\operatorname{ker}\left(\alpha_{x_{i}}\right) \in \mathbf{T}$. Therefore, $\operatorname{ker}\left(\alpha_{x}\right) \in \mathbf{T}$ by T2. Hence $\mathbf{t}\left(M_{1} \times \ldots \times M_{n}\right)=$ $M_{1} \times \ldots \times M_{n}$.

THEOREM 4.4. The set of hereditary pretorsion classes of $S$-automata can also be parametrized by the set of idempotent preradicals $\mathbf{r}$ such that $\mathbf{C}_{\mathbf{r}}=\{M \mid M$ is an $S$-automaton and $\mathbf{r}(M)=M\}$ is closed under subautomata and finite products.

Proof. Let $\mathbf{C}$ be an HPC. Define $\mathbf{T}$ by

$$
\mathbf{T}=\left\{\rho \mid \rho \text { is a right congruence on } S^{1} \text { and } S^{1} / \rho \in \mathbf{C}\right\} .
$$

Then define $\mathbf{t}$ on $\operatorname{Aut}(S)$ as $\mathbf{t}(M)=\left\{x \in M \mid \operatorname{ker}\left(\alpha_{x}\right) \in \mathbf{T}\right\}$ and $\mathbf{C}_{\mathbf{t}}=\{M \mid \mathbf{t}(M)=M\}$. Suppose $M \in \mathbf{C}_{\mathbf{t}}$, then $\mathbf{t}(M)=M$ which implies that $\operatorname{ker}\left(\alpha_{x}\right) \in \mathbf{T}, \forall x \in M$. Then $x S^{1} \cong$ $S^{1} / \operatorname{ker}\left(\alpha_{x}\right) \in \mathbf{C}$ for all $x \in M$ by definition of $\mathbf{T}$. So $\oplus x S^{1} \in \mathbf{C}$ and hence $M$, being the homomorphic image of $\oplus x S^{1}$, belongs to $\mathbf{C}$. On the other hand, if $M \in \mathbf{C}$, then $\operatorname{ker}\left(\alpha_{x}\right) \in \mathbf{T}, \forall x \in M$ by the proof of Theorem 3.3. Then $\mathbf{t}(M)=M$ and $M \in \mathbf{C}_{\mathbf{t}}$. Hence $\mathbf{C}=\mathbf{C}_{\mathbf{t}}$.

Conversely, let $\mathbf{t}$ be an idempotent preradical on $\operatorname{Aut}(S)$ such that $\mathbf{C}_{\mathbf{t}}$ is closed under subautomata and finite products. Then $\mathbf{C}_{\mathbf{t}}$ is an HPC because the proof in Theorem 4.3 on closure under quotients and coproducts does not depend on the particular definition of t. By Theorem 3.3, we get an RL-topology

$$
\mathbf{T}=\left\{\rho \mid \rho \text { is a right congruence on } S^{1} \text { and } S^{1} / \rho \in \mathbf{C}_{\mathbf{t}}\right\}
$$


We define $\mathbf{t}_{1}$ on $\operatorname{Aut}(S)$ by $\mathbf{t}_{\mathbf{1}}(M)=\left\{x \in M \mid \operatorname{ker}\left(\alpha_{x}\right) \in \mathbf{T}\right\}$. We would like to show that $\mathbf{t}_{\mathbf{1}}=\mathbf{t}$. Suppose $M \in \operatorname{Aut}(S)$. Let $\boldsymbol{x} \in \mathbf{t}_{\mathbf{1}}(M)$; then $\operatorname{ker}\left(\alpha_{x}\right) \in \mathbf{T}$ which implies that $S^{1} / \operatorname{ker}\left(\alpha_{x}\right) \in \mathbf{C}_{\mathbf{t}}$. Then $x S^{1} \in \mathbf{C}_{\mathbf{1}}$. Now $\oplus x S^{1} \in \mathbf{C}_{\mathbf{1}}$ and so is $\mathbf{t}_{\mathbf{1}}(M)$. Therefore $\mathbf{t}\left(\mathbf{t}_{\mathbf{1}}(M)\right)=$ $\mathbf{t}_{\mathbf{1}}(M)$. But $M \supset \mathbf{t}_{1}(M)$; so $\mathbf{t}(M) \supset \mathbf{t}_{1}(M)$. On the other hand, we show that $\mathbf{t}_{1}(M)$ is the largest subautomaton of $M$ belonging to $\mathbf{C}_{\mathbf{t}}$. Suppose $M \supset N \supset \mathbf{t}_{\mathbf{l}}(M)$ and $N \in \mathbf{C}_{\mathbf{t}}$. Let $x \in N \backslash \mathbf{t}_{1}(M)$; then $\operatorname{ker}\left(\alpha_{x}\right) \notin \mathbf{T}$ and thus $x S^{1} \cong S^{1} / \operatorname{ker}\left(\alpha_{x}\right) \notin \mathbf{C}_{\mathbf{t}}$. Since $N \in \mathbf{C}_{\mathbf{t}}$ and $x S^{1}$ is a subautomaton of $N, x S^{1} \in \mathbf{C}_{\mathbf{t}}$. So we have reached a contradiction. Furthermore, $\mathbf{t}(M) \in \mathbf{C}_{\mathbf{1}}$ since $\mathbf{t}$ is idempotent. We then have $\mathbf{t}_{\mathbf{1}}(M) \supset \mathbf{t}(M)$. Hence $\mathbf{t}_{\mathbf{1}}=\mathbf{t}$.

5. Semigroups of fractions and automata of fractions. We summarize here the previous results from Sections 3 and 4 as follows.

THEOREM 5.1. The set of hereditary pretorsion classes of S-automata can be parameterized by both the set of right linear topologies on $S^{1}$ and the set of idempotent preradicals $\mathbf{t}$ on Aut $(S)$ such that $\mathbf{C}_{\mathrm{t}}=\{M / \mathbf{t}(M)=M\}$ is closed under subautomata and finite products.

Next, we turn our attention to the concepts of semigroups of fractions and automata of fractions. Then we construct a specific example for an HPC and use the Correspondence Theorem 5.1 to develop some interesting results. We first present some preliminary definitions and results while details and proofs may be found in $[\mathbf{1}, \mathbf{4}, \mathbf{5}]$.

Definition 5.1. Let $S$ be a semigroup as always and $A$ be a subsemigroup. A right semigroup of fractions is a monoid $S\left[A^{-1}\right]$ together with a semigroup homomorphism $\phi: S \rightarrow S\left[A^{-1}\right]$ satisfying the following conditions.

S1. $\phi(a)$ is invertible for all $a \in A$ with respect to the identity.

S2. Every element in $S\left[A^{-1}\right]$ has the form $\phi(s) \phi(a)^{-1}$ with $s \in S$ and $a \in A$.

S3. $\phi(s)=\phi(t)$ if and only if $s a=t a$ for some $a \in A$.

LEMmA 5.1. $S\left[A^{-1}\right]$ exists if and only if the subsemigroup $A$ satisfies:

A1. If $a \in A$ and $s \in S$, then there exists $b \in A$ and $t \in S$ such that $a t=s b$.

A2. If $a \in A$ and $a s=a t$ with $s, t \in S$, then there exists $b \in A$ such that $s b=t b$.

LEMMA 5.2. When $S\left[A^{-1}\right]$ exists, it has the following universal property: for every semigroup homomorphism $\lambda: S \rightarrow T$ such that $\lambda(a)$ is invertible in $T$, for all $a \in A$, there exists a unique homomorphism $\sigma: s\left[A^{-1}\right] \rightarrow T$ such that $\sigma \circ \phi=\lambda$. We then conclude that $S\left[A^{-1}\right]$ is unique up to isomorphism.

When $S\left[A^{-1}\right]$ exists, it has the form $S\left[A^{-1}\right]=S \times A / \sim$ where $\sim$ is the equivalence relation defined on $S \times A$ as $(s, a) \sim(t, b)$ if there exist $u, v \in S$ such that $s u=t v$ and $a u=b v \in A$. If we denote elements of $S\left[A^{-1}\right]$ by $[s, a]$, then $\phi: S \rightarrow S\left[A^{-1}\right]$ is defined as $\phi(s)=[s a, a]$ for any $a \in A$. Moreover, it is easy to show that the identity in $S\left[A^{-1}\right]$ is $[a, a]$ for any $a \in A$.

Definition 5.2. A subsemigroup $A$ of $S$ is called a right denominator set when it satisfies $\mathrm{A} 1$ and $\mathrm{A} 2$.

Definition 5.3. Suppose $S\left[A^{-1}\right]$ exists and $M$ is an $S$-automaton. An automaton of fractions with respect to $A$ is an $S\left[A^{-1}\right]$-automaton $M\left[A^{-1}\right]$. 
It has the form $M\left[A^{-1}\right]=M \times A / \sim$, where $\sim$ is the equivalence relation defined on $M \times A$ as $(m, a) \sim(n, b)$ if there exist $s, t \in S$ such that $m s=n t$ and $a s=b t \in A$. We denote elements of $M\left[A^{-1}\right]$ by $[m, a]$. Moreover, $S\left[A^{-1}\right]$ operates on $M\left[A^{-1}\right]$ by $[m, a][s, b]=[m t, b c]$, where $[s, b] \in S\left[A^{-1}\right], t \in S, c \in A$ and $a t=s c$.

LEMMA 5.3. Suppose $A$ is a denominator set. If $N$ is an $S\left[A^{-1}\right]$-automaton, then $N$ is an $S$-automaton with the $S$-operation defined by $n . s=n \phi(s)$, where $\phi$ is as defined in Definition 5.1.

LEMMA 5.4. Suppose that $M\left[A^{-1}\right]$ is an $S\left[A^{-1}\right]$-automaton. Then the function $\mu_{M}: M \rightarrow\left[A^{-1}\right]$, defined by $\mu_{M}(m)=[m a, a]$ for any $a \in A$ is a well-defined $S$ homomorphism. Moreover $\mu_{M}$ satisfies the following universal property: for each $S\left[A^{-1}\right]$-automaton $N$ and $S$-homomorphism $\alpha: M \rightarrow N$ with the $S$-action as defined in Lemma 5.3, there exists a unique $S\left[A^{-1}\right]$-homomorphism $\sigma: M\left[A^{-1}\right] \rightarrow N$ such that $\sigma \mu_{M}=\alpha$. Moreover, each element $[m, a] \in M\left[A^{-1}\right]$ can be expressed as $\mu_{M}(m) \phi(a)^{-1}$.

Let $\mu_{M}: M \rightarrow M\left[A^{-1}\right]$ be defined as above.

Definition 5.4. $\operatorname{ker}\left(\mu_{M}\right)$ is called the $A$-torsion of $M$.

Lemma 5.5. $\operatorname{ker}\left(\mu_{M}\right)=\{(m, n) \in M \times M / \exists c \in A(m c=n c)\}$.

Definition 5.5. $M$ is an $A$-torsion $S$-automaton if $M$ is an $S$-automaton and $\operatorname{ker}\left(\mu_{M}\right)$ is the universal congruence on $M . M$ is $A$-torsion-free if $\operatorname{ker}\left(\mu_{M}\right)$ is the trivial congruence.

TheOREM 5.2. Let $\mathbf{C}$ be the set of A-torsion $S$-automata and their coproducts. Then $\mathbf{C}$ is an HPC.

Proof. We show that $\mathbf{C}$ is closed under subautomata, quotients, coproducts and finite products. It is clear that subautomata of $A$-torsion $S$-automata are $A$-torsion by using the definition of $\operatorname{ker}\left(\mu_{M}\right)$. Suppose $\left\{M_{\alpha}\right\}$ is a set of $A$-torsion $S$-automata and $N$ is a subautomaton of $\oplus M_{\alpha}$. Then since $\oplus M_{\alpha}$ is a disjoint union $N=\oplus N_{\alpha}$, where $N_{\alpha}=$ $N \cap M_{\alpha}, \forall \alpha$. It is clear that each $N_{\alpha}$ is an $S$-subautomaton of $M_{\alpha}$ and thus is $A$-torsion. Hence $N$ is a coproduct of $A$-torsion $S$-automata.

Next, suppose $M$ is $A$-torsion and $\rho$ is a right congruence of $M$. Let $\hat{M}=M / \rho$. Consider the map $\mu_{\hat{M}}: \hat{M} \rightarrow \hat{M}\left[A^{-1}\right]$ defined as before. By Lemma $5.5, \operatorname{ker}\left(\mu_{\hat{M}}\right)=$ $\{([m],[n]) \in \hat{M} \times \hat{M} \mid \exists c \in A([m] c=[n] c)\}$. Since $M$ is $A$-torsion, if $m, n \in M$, then there exists $c \in A$ such that $m c=n c$. Then $[m] c=[n] c$. Thus $([m],[n]) \in \operatorname{ker}\left(\mu_{\hat{M}}\right)$. We then conclude that $M / \rho$ is also $A$-torsion. Now suppose $\left\{M_{\alpha}\right\}$ is a set of $A$-torsion $S$-automata and $\rho$ is a right congruence on $\bigoplus M_{\alpha}$. Let $\mathbf{D}=\left\{\mathbf{D}_{j}\right\}$ be the set of equivalence classes of $\rho$. For each $\alpha$, let $E_{\alpha}=\left\{\mathbf{D}_{j} \mid \mathbf{D}_{j} \cap M_{\alpha} \neq \varnothing\right\}$. We claim that $E_{\alpha}$ is an $A$-torsion $S$-automaton for each $\alpha$. First of all, $E_{\alpha} \neq \varnothing$. Assume not, then there is an $\alpha$ such that $\mathbf{D}_{j} \cap M_{\alpha}=\varnothing$ for all $j$. Then $\cup \mathbf{D}_{j}=\left(\bigcup M_{\beta}\right) \backslash M_{\alpha}$ which is absurd. Next, suppose $\mathbf{D}_{j} \in E_{\alpha}$ and $s \in S$; then $\mathbf{D}_{j} \cap M_{\alpha} \neq \varnothing$. So there is $x \in \mathbf{D}_{j} \cap M_{\alpha}$, and $x s \in \mathbf{D}_{j} s \cap M_{\alpha}$. This implies that $\mathbf{D}_{j} s \in E_{\alpha}$ and so $E_{\alpha}$ is an $S$-automaton. Suppose $\mathbf{D}_{j}$ and $\mathbf{D}_{k}$ are arbitrary elements of $E_{\alpha}$; then $\mathbf{D}_{j} \cap M_{\alpha} \neq \varnothing$ and $\mathbf{D}_{k} \cap M_{\alpha} \neq \varnothing$. Let $d_{j} \in \mathbf{D}_{j} \cap M_{\alpha}$ and $d_{k} \in \mathbf{D}_{k} \cap M_{\alpha}$. By hypothesis, $M_{\alpha}$ is $A$-torsion, and so there is $c \in A$ such that $d_{j} c=d_{k} c$. Then $\mathbf{D}_{j} c=\mathbf{D}_{k} c$ and $\left(\mathbf{D}_{j}, \mathbf{D}_{k}\right) \in$ $\operatorname{ker}\left(\mu_{E_{\mathrm{n}}}\right)$. Hence, we conclude that $\operatorname{ker}\left(\mu_{E_{\mathrm{a}}}\right)$ is the universal congruence. We then have $\bigoplus E_{\alpha} \in \mathbf{C}$. Since $\mathbf{D}$ is the homomorphic image of $\oplus E_{\alpha}$ under the $S$-homomorphism which forgets the extra separating indices from the same elements $\mathbf{D}_{j}$ but in different automata $E_{\alpha}, \oplus M_{\alpha} / \rho=\mathbf{D} \in \mathbf{C}$. Hence, $\mathbf{C}$ is closed under quotients. 
Finally, we show that $\mathbf{C}$ is closed under finite products. We first show that the finite product of $A$-torsion $S$-automata is $A$-torsion. However, it suffices to show that it is true for only two $A$-torsion $S$-automata, say, $M$ and $N$. Suppose $(m, n)$ and $(x, y)$ are arbitrary elements of $M \times N$. Since $M$ and $N$ are $A$-torsion, there exist $c, d \in A$ such that $m c=x c$ and $n d=y d$. Moreover, $c \in A$ and $d \in S$ imply that there exist $b \in A$ and $e \in S$ such that $c e=d b$ by A1 in Lemma 5.2. Therefore, $m c e=x c e$ and $n d b=y d b$. Let $g=d b \in A$; then $m g=x g$ and $n g=y g$. We then conclude that $\operatorname{ker}\left(\mu_{M \times N}\right)$ is the universal congruence and $M \times N$ is $A$-torsion. Next, suppose $\oplus M_{\alpha}$ and $\oplus N_{\beta}$ are coproducts of $A$-torsion $S$-automata. Then it is easy to show that $\left(\oplus M_{\alpha}\right) \times\left(\oplus N_{\beta}\right) \cong \oplus\left(M_{\alpha} \times N_{\beta}\right)$. Since $M_{\alpha} \times N_{\beta}$ is $A$-torsion by the proof above, $\left(\oplus M_{\alpha}\right) \times\left(\oplus N_{\beta}\right)$ is a coproduct of $A$-torsion $S$-automata.

Corollary 5.1. Let $\mathbf{C}$ be the set of A-torsion S-automata and their coproducts; then $\mathbf{C}$ is the set of $S$-automata each cyclic subautomaton of which is A-torsion.

Proof. Since $\mathbf{C}$ is an HPC by Theorem 5.2, we can define

$$
\mathbf{T}=\left\{\rho \mid \rho \text { is a right congruence on } S^{1} \text { and } S^{1} / \rho \in \mathbf{C}\right\}
$$

by the Correspondence Theorem 5.1. Then

$$
\mathbf{C}=\left\{M \mid M \text { is an } S \text {-automaton and } \operatorname{ker}\left(\alpha_{x}\right) \in \mathbf{T}, \forall x \in M\right\}
$$

by Theorem 5.1 again. However, $\operatorname{ker}\left(\alpha_{x}\right) \in \mathbf{T}, \forall x \in M$ if and only if $S^{1} / \operatorname{ker}\left(\alpha_{x}\right) \in \mathbf{C}, \forall x \in$ $M$. This is equivalent to $x S^{1} \in \mathbf{C}, \forall x \in M$, which is then equivalent to $x S^{1}$ being $A$-torsion for all $x \in M$.

Definition 5.6. Elements of $\mathbf{C}$, as defined in Corollary 5.1, are called pre-A-torsion $S$-automata.

$A$-torsion $S$-automata are obviously also pre- $A$-torsion. Moreover, pre- $A$-torsion $S$-automata are pretorsion since $\mathbf{C}$ is an HPC.

Notice that

$$
\begin{aligned}
\mathbf{C} & =\{M \mid M \text { is an } S \text {-automaton and each cyclic subautomaton is } A \text {-torsion }\} \\
& =\left\{M \mid x S^{1} \text { is } A \text {-torsion for all } x \in M\right\} \\
& =\left\{M \mid \operatorname{ker}\left(\mu_{x s^{\prime}}\right)=\text { universal congruence for all } x \in M\right\} .
\end{aligned}
$$

We define $\mathbf{t}_{2}$ on $\operatorname{Aut}(S)$ as

$$
\mathbf{t}_{\mathbf{2}}(M)=\left\{x \in M \mid \operatorname{ker}\left(\mu_{x s^{\prime}}\right)=\text { universal congurence }\right\} .
$$

Then $\mathbf{C}=\left\{M \mid \mathbf{t}_{\mathbf{2}}(M)=M\right\}$. Since $\mathbf{C}$ is an HPC, we can define, via the Correspondence Theorem 5.1, an RL-topology

$$
\mathbf{T}=\left\{\rho \mid \rho \text { is a right congruence on } S^{1} \text { and } S^{1} / \rho \in \mathbf{C}\right\} .
$$

Then we get our original $\mathbf{t}$ which is defined as $\mathbf{t}(M)=\left\{x \in M \mid \operatorname{ker}\left(\alpha_{x}\right) \in \mathbf{T}\right\}$.

Corollary 5.2. $\mathbf{t}_{\mathbf{2}}=\mathbf{t}$.

Proof. $x \in \mathbf{t}(M)$ if and only if $\operatorname{ker}\left(\alpha_{x}\right) \in \mathbf{T}$. Then this is equivalent to $S^{1} / \operatorname{ker}\left(\alpha_{x}\right) \in \mathbf{C}$. Then $x S^{1} \in \mathbf{C}$ since $S^{1} / \operatorname{ker}\left(\alpha_{x}\right) \cong x S^{1}$. But this is equivalent to $\operatorname{ker}\left(\mu_{x S^{1}}\right)=$ universal congruence and it is the same as $x \in \mathbf{t}_{\mathbf{2}}(M)$. Hence $\mathbf{t}_{\mathbf{2}}$ is also an idempotent preradical such that $\mathbf{C}=\left\{M \mid \mathbf{t}_{\mathbf{2}}(M)=M\right\}$ is closed under subautomata and finite products. 


\section{REFERENCES}

1. C. S. Lam and R. H. Oehmke, Some results on automata, to be published in the Proceedings of the Second International Conference on Languages, Words, and Semigroups, Kyoto, Japan, 1992.

2. J. K. Luedeman, Torsion theories and semigroups of quotients, in Lecture Notes in Mathematics No. 998, (Springer-Verlag, 1983), 350-373.

3. L. Marki, R. Mlitz, and R. Strecker, Strict radicals of monoids, Semigroup Forum 21 (1980), 27-66.

4. B. Stenstrom, Rings of quotients (Springer-Verlag, 1975).

5. X. D. Ye, Semigroups of quotients, Ph.D. Thesis (University of Iowa, lowa City, Iowa, 1987).

Department of Mathematics

Ithaca College

ITHACA, NY 14850

U.S.A. 